\title{
Article
}

\section{Risk of Aortic Aneurysm and Dissection in Patients with Tuberculosis: A Nationwide Population-Based Cohort Study}

\author{
Ming-Tsung Chen ${ }^{1}(\mathbb{D})$, Chi-Hsiang Chung ${ }^{2,3}{ }^{\mathbb{D}}$, Hung-Yen $\mathrm{Ke}^{4}$, Chung-Kan Peng ${ }^{1}$ (D), Wu-Chien Chien ${ }^{2,3, *(\mathbb{D})}$ \\ and Chih-Hao Shen $1, *$ (D)
}

check for

updates

Citation: Chen, M.-T.; Chung, C.-H.; Ke, H.-Y.; Peng, C.-K.; Chien, W.-C.;

Shen, C.-H. Risk of Aortic Aneurysm and Dissection in Patients with

Tuberculosis: A Nationwide

Population-Based Cohort Study. Int. J.

Environ. Res. Public Health 2021, 18 ,

11075. https://doi.org/10.3390/

ijerph182111075

Academic Editor: Paul B. Tchounwou

Received: 10 August 2021

Accepted: 19 October 2021

Published: 21 October 2021

Publisher's Note: MDPI stays neutral with regard to jurisdictional claims in published maps and institutional affiliations.

Copyright: (c) 2021 by the authors. Licensee MDPI, Basel, Switzerland. This article is an open access article distributed under the terms and conditions of the Creative Commons Attribution (CC BY) license (https:/ / creativecommons.org/licenses/by/ $4.0 /)$.
1 Division of Pulmonary and Critical Care Medicine, Department of Internal Medicine, Tri-Service General Hospital, National Defense Medical Center, Taipei 11490, Taiwan; a01016233@yahoo.com.tw (M.-T.C.); kanpeng1025@yahoo.com.tw (C.-K.P.)

2 Department of Medical Research, Tri-Service General Hospital, National Defense Medical Center, Taipei 11490, Taiwan; g694810042@gmail.com

3 School of Public Health, National Defense Medical Center, Taipei 11490, Taiwan

4 Division of Cardiovascular Surgery, Department of Surgery, Tri-Service General Hospital, National Defense Medical Center, Taipei 11490, Taiwan; drkehy@yahoo.com.tw

* Correspondence: chienwu@mail.ndmctsgh.edu.tw (W.-C.C.); potato652@yahoo.com.tw (C.-H.S.); Tel.: +886-2-87923311 (W.-C.C. \& C.-H.S.)

\begin{abstract}
Tuberculosis (TB) can cause chronic inflammation. The occurrence of aortic aneurysm (AA) and aortic dissection (AD) may be associated with chronic inflammatory disease, but whether TB increases the risk of $\mathrm{AA}$ and $\mathrm{AD}$ remains to be determined. This study aimed to investigate the association between TB and the development of AA and AD. We conducted a population-based cohort study using data obtained from the Taiwan National Health Insurance Database. We selected 31,220 individuals with TB and 62,440 individuals without TB by matching the cohorts according to age, sex, and index year at a ratio of 1:2. Cox regression analysis revealed that the TB cohort had a 1.711-fold higher risk of $\mathrm{AA}$ and $\mathrm{AD}$ than the non-TB cohort after adjustment for sex, age, socioeconomic status, and comorbidities (adjusted hazard ratio $=1.711 ; 95 \%$ confidence interval $=1.098-2.666$ ). Patients with pulmonary, extrapulmonary, and miliary TB had a 1.561-, 1.892-, and 8.334-fold higher risk of AA and AD, respectively. Furthermore, patients with TB at $<6$ months, $6-12$ months, and $1-5$ years of follow-up had a 6.896-, 2.671-, and 2.371-fold risk of AA and AD, respectively. Physicians should consider the subsequent development of $\mathrm{AA}$ and $\mathrm{AD}$ while treating patients with $\mathrm{TB}$.
\end{abstract}

Keywords: tuberculosis; aortic aneurysm; aortic dissection

\section{Introduction}

Tuberculosis (TB), an infectious disease caused by Mycobacterium tuberculosis, is one of the major causes of death worldwide. In 2019, approximately 10 million individuals were diagnosed with TB, and 1.4 million individuals died because of TB [1]. In Taiwan, the incidence of TB was 37 cases per 100,000 people and that of TB-related deaths was 2.3 per 100,000 people in 2019 [2]. TB typically affects the lungs and results in cavitation, fibrosis, bronchiectasis, and impaired pulmonary function [3]. TB that affects other organs, such as the lymph nodes, pleura, gastrointestinal tract, bones, urogenital tract, and central nervous system, is known as extrapulmonary TB and accounts for $20-25 \%$ of all TB cases [4].

Aortic aneurysm (AA) is the permanent dilation of the aorta and most commonly occurs in the infrarenal and proximal thoracic aortic regions. Most AAs are asymptomatic, but progressive enlargement of the aneurysm increases the risk of dissection and rupture [5]. Aortic dissection (AD) is defined as a tear in the inner layer of the aortic wall that leads to the formation of true and false lumens. AD is a relatively uncommon, life-threatening vascular disease with an annual incidence ranging from 3 to 6 cases per 100,000 persons [6,7]. AA and AD have common risk factors, including smoking, hypertension (HTN), genetic connective 
tissue disorders, male sex, older age, and vascular inflammation caused by infection or atherosclerosis [6,8-10]. Infection can lead to arterial wall aneurysmal degeneration, referred to as mycotic aneurysm, a term first coined by William Osler in 1885 [11], and constitutes $0.6-2 \%$ of all arterial aneurysms. A review of the literature on mycotic AA from 2000 to 2018 found that the most common microorganism isolated in the disease was Salmonella spp. (33.4\%), followed by Staphylococcus spp. (15.6\%), Streptococcus spp. (10.4\%), and Escherichia coli (3.1\%). However, Mycobacteria spp. only constituted 2\% of all cases [12].

Previous studies demonstrated that patients with TB have a higher risk of developing systemic diseases such as liver cirrhosis, Parkinson's disease, sarcoidosis, systemic lupus erythematosus, acute coronary syndrome, and peripheral arterial disease [13-18]. Inflammation caused by immune response activation and cytokine induction is a crucial pathogenic mechanism of TB infection [19]. Chronic inflammation also plays an essential role in atherosclerosis, which leads to the development of various cardiovascular diseases $[20,21]$. Because chronic inflammation increases the risk of developing AA and $\mathrm{AD}[22,23]$, and as TB may cause chronic inflammation, we hypothesized that patients with $\mathrm{TB}$ have an increased risk of developing $\mathrm{AA}$ and $\mathrm{AD}$. We conducted this nationwide population-based cohort study to assess the association between these two diseases.

\section{Materials and Methods}

\subsection{Data Source}

The data assessed in this study were obtained from the Longitudinal Health Insurance Database (LHID), a subset of the Taiwan National Health Insurance (NHI) Research Database (NHIRD). NHIRD maintains data on all claims of the beneficiaries of the Taiwan NHI program and uses the International Classification of Diseases, Ninth Revision, Clinical Modification (ICD-9-CM) system for recording diagnoses. The Taiwanese government established the NHI in 1995 as a single-payer compulsory program for all 23 million Taiwanese people. For the protection of privacy, NHIRD removes identifying information and assigns an anonymous number before releasing patient records for research purposes. This study was approved by the Institutional Review Board of the Tri-Service General Hospital, National Defense Medical Center (TSGHIRB No. B-110-21).

\subsection{Sampled Patients}

We conducted a nationwide population-based cohort study to investigate the incidence of $\mathrm{AA}$ and $\mathrm{AD}$ among patients with $\mathrm{TB}$ and identify the associated risk factors. Records of patients aged $\geq 20$ years who were diagnosed with TB (ICD-9-CM 010-018) between 2000 and 2015 were obtained from the LHID (Figure 1). The date of diagnosis served as the index date. The follow-up period was defined as the interval from the index date to the date of $\mathrm{AA}$ and $\mathrm{AD}$ diagnosis. We excluded patients with a history of TB, AA, or AD (ICD-9-CM 441.0-441.9) before the index date, those aged $<20$ years, and those with incomplete medical information. We randomly selected the TB and non-TB cohorts with the same exclusion criteria from the LHID and matched them by frequency according to their age, sex, and index year at a ratio of 1:2. The TB cohort was subdivided into pulmonary, extrapulmonary, and miliary groups for subgroup analyses. Furthermore, the TB cohort was classified according to different sites of AA and AD, namely, thoracic (ICD9-CM 441.01, 441.1, 441.2), abdominal (ICD-9-CM 441.02, 441.3, 441.4), thoracoabdominal (ICD-9-CM 441.03, 441.6, 441.7), and unspecified sites (ICD-9-CM 441.00, 441.5, 441.9).

\subsection{Outcome Measurement and Comorbidities}

All the patients were followed up from the index date until the first diagnosis of AA/AD, death, withdrawal from the NHI program, or 31 December 2015. The study included baseline comorbidities such as diabetes mellitus (DM; ICD-9-CM 250), HTN (ICD9-CM 401-405), hyperlipidemia (ICD-9-CM 272), ischemic heart disease (IHD; ICD-9-CM 410-414), chronic obstructive pulmonary disease (COPD; ICD-9-CM 490-496), stroke (ICD- 
9-CM 438), chronic kidney disease (CKD; ICD-9-CM 585), peripheral arterial occlusion disease (PAOD; ICD-9-CM 443.9), and obesity (ICD-9-CM 278.0-278.1).

\section{Outpatient and inpatient of Longitudinal Health Insurance Database in 2000-2015 in Taiwan}

$36,464,228$ events; $1,936,512$ individuals

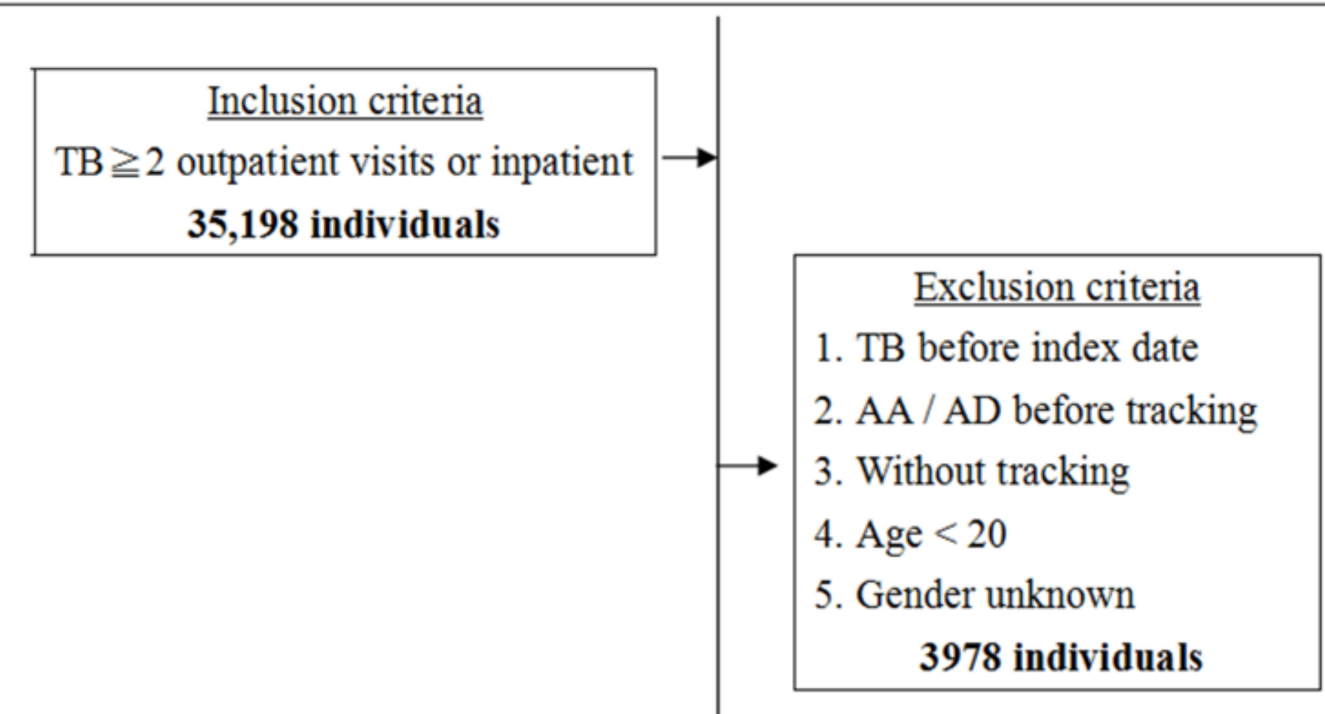

1. Without TB in study period

2. The same exclusion criteria of study cohort

3. 2-fold propensity score matching by gender, age, index date
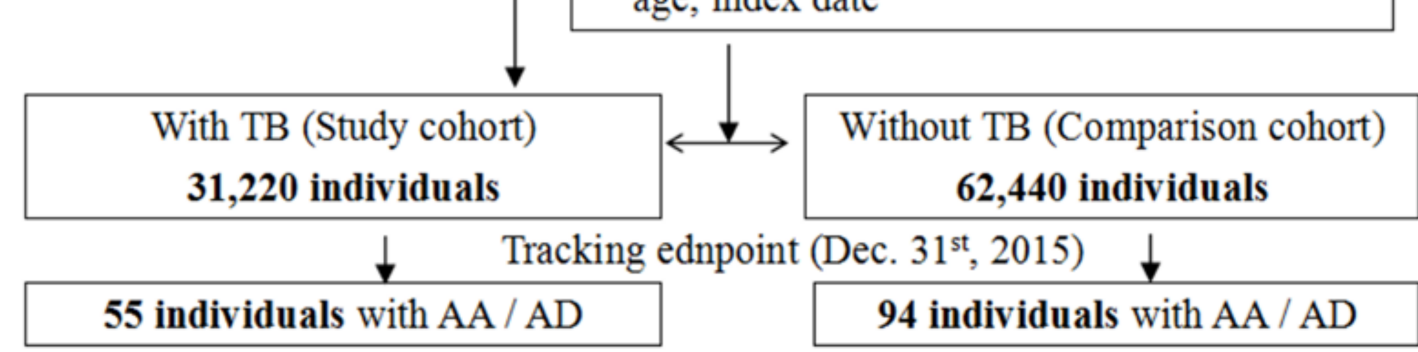

Figure 1. Patient selection flowchart. $\mathrm{TB}=$ tuberculosis, $\mathrm{AA}=$ aortic aneurysm, $\mathrm{AD}=$ aortic dissection.

\subsection{Statistical Analysis}

We compared the distribution of categorical characteristics and baseline comorbidities between patients with and without TB using the chi-square test. In addition, we compared continuous variables between the cohorts using Student's $t$-test. We used the KaplanMeier method to estimate the cumulative incidences of $\mathrm{AA}$ and $\mathrm{AD}$ and performed the log-rank test to examine the differences between the cohorts. The incidence rate ratios (IRR) of $\mathrm{AA}$ and $\mathrm{AD}$ in both cohorts were estimated using Poisson regression analysis. Cox regression analysis was used to estimate the adjusted hazard ratios (HR) for the development of AA and AD after adjusting for sex, age, insured premium, urbanization level, and comorbidities, such as DM, HTN, hyperlipidemia, IHD, COPD, stroke, CKD, PAOD, and obesity. All the analyses were conducted using SPSS software, version 26 (SPSS Inc., Chicago, IL, USA). $p$-values $<0.05$ indicated statistical significance for a two-sided test. 


\section{Results}

We included 31,220 patients in the TB cohort and 62,440 patients in the non-TB cohort. The distributions of age and sex were similar between the cohorts. The mean followup periods for the TB and non-TB cohorts were $9.52 \pm 11.02$ and $10.04 \pm 9.18$ years, respectively. The patients were predominantly male $(71.17 \%)$ and aged $\geq 70$ years $(46.41 \%)$. The TB cohort had a higher prevalence of DM and COPD; lower prevalence of HTN, hyperlipidemia, IHD, stroke, CKD, and obesity; and a lower urbanization level than the non-TB cohort (Table 1).

Table 1. Characteristics of the study participants at baseline.

\begin{tabular}{|c|c|c|c|c|c|c|c|}
\hline \multirow[b]{2}{*}{ Variables } & \multicolumn{2}{|c|}{ Total } & \multicolumn{2}{|c|}{ With TB } & \multicolumn{2}{|c|}{ Without TB } & \multirow[b]{2}{*}{$p$} \\
\hline & $\mathbf{n}$ & $\%$ & $\mathbf{n}$ & $\%$ & $\mathrm{n}$ & $\%$ & \\
\hline Overall & 93,660 & & 31,220 & 33.33 & 62,440 & 66.67 & \\
\hline Sex & & & & & & & 0.999 \\
\hline Male & 66,657 & 71.17 & 22,219 & 71.17 & 44,438 & 71.17 & \\
\hline Female & 27,003 & 28.83 & 9001 & 28.83 & 18,002 & 28.83 & \\
\hline Age (years) & \multicolumn{2}{|c|}{$63.67 \pm 17.32$} & \multicolumn{2}{|c|}{$63.74 \pm 17.33$} & \multicolumn{2}{|c|}{$63.64 \pm 17.32$} & 0.405 \\
\hline Age group (years) & & & & & & & 0.999 \\
\hline $20-44$ & 16,314 & 17.42 & 5438 & 17.42 & 10,876 & 17.42 & \\
\hline $45-69$ & 33,876 & 36.17 & 11,292 & 36.17 & 22,584 & 36.17 & \\
\hline$\geq 70$ & 43,470 & 46.41 & 14,490 & 46.41 & 28,980 & 46.41 & \\
\hline $\begin{array}{c}\text { Insured premium } \\
(\mathrm{NT} \$)\end{array}$ & & & & & & & 0.003 \\
\hline$<18,000$ & 92,378 & 98.63 & 30,768 & 98.55 & 61,610 & 98.67 & \\
\hline $18,000-34,999$ & 1032 & 1.10 & 385 & 1.23 & 647 & 1.04 & \\
\hline$\geq 35,000$ & 250 & 0.27 & 67 & 0.21 & 183 & 0.29 & \\
\hline $\mathrm{DM}$ & & & & & & & $<0.001$ \\
\hline Without & 70,368 & 75.13 & 23,042 & 73.81 & 47,326 & 75.79 & \\
\hline With & 23,292 & 24.87 & 8178 & 26.19 & 15,114 & 24.21 & \\
\hline HTN & & & & & & & $<0.001$ \\
\hline Without & 59,003 & 63.00 & 21,732 & 69.61 & 37,271 & 59.69 & \\
\hline With & 34,657 & 37.00 & 9488 & 30.39 & 25,169 & 40.31 & \\
\hline Hyperlipidemia & & & & & & & $<0.001$ \\
\hline Without & 87,769 & 93.71 & 30,159 & 96.60 & 57,610 & 92.26 & \\
\hline With & 5891 & 6.29 & 1061 & 3.40 & 4830 & 7.74 & \\
\hline IHD & & & & & & & $<0.001$ \\
\hline Without & 71,662 & 76.51 & 26,219 & 83.98 & 45,443 & 72.78 & \\
\hline With & 21,998 & 23.49 & 5001 & 16.02 & 16,997 & 27.22 & \\
\hline COPD & & & & & & & $<0.001$ \\
\hline Without & 69,531 & 74.24 & 21,719 & 69.57 & 47,812 & 76.57 & \\
\hline With & 24,129 & 25.76 & 9501 & 30.43 & 14,628 & 23.43 & \\
\hline Stroke & & & & & & & $<0.001$ \\
\hline Without & 73,991 & 79.00 & 26,519 & 84.94 & 47,472 & 76.03 & \\
\hline With & 19,669 & 21.00 & 4701 & 15.06 & 14,968 & 23.97 & \\
\hline CKD & & & & & & & $<0.001$ \\
\hline Without & 89,710 & 95.78 & 30,153 & 96.58 & 59,557 & 95.38 & \\
\hline With & 3950 & 4.22 & 1067 & 3.42 & 2883 & 4.62 & \\
\hline PAOD & & & & & & & 0.053 \\
\hline Without & 93,603 & 99.94 & 31,208 & 99.96 & 62,395 & 99.93 & \\
\hline With & 57 & 0.06 & 12 & 0.04 & 45 & 0.07 & \\
\hline Obesity & & & & & & & 0.002 \\
\hline Without & 93,632 & 99.97 & 31,218 & 99.99 & 62,414 & 99.96 & \\
\hline With & 28 & 0.03 & 2 & 0.01 & 26 & 0.04 & \\
\hline Urbanization level & & & & & & & $<0.001$ \\
\hline 1 (The highest) & 28,768 & 30.72 & 8960 & 28.70 & 19,808 & 31.72 & \\
\hline 2 & 41,696 & 44.52 & 13,732 & 43.98 & 27,964 & 44.79 & \\
\hline 3 & 7425 & 7.93 & 2565 & 8.22 & 4860 & 7.78 & \\
\hline 4 (The lowest) & 15,771 & 16.84 & 5963 & 19.10 & 9808 & 15.71 & \\
\hline
\end{tabular}

$\mathrm{DM}=$ diabetes mellitus, $\mathrm{HTN}=$ hypertension, $\mathrm{IHD}=$ ischemic heart disease, $\mathrm{COPD}=$ chronic obstructive pulmonary disease, $\mathrm{CKD}=$ chronic kidney disease, $\mathrm{PAOD}=$ peripheral arterial occlusive disease. 
Patients with TB had a higher risk for further development of AA and AD than patients without TB (adjusted HR $=1.711 ; 95 \% \mathrm{CI}=1.098-2.666 ; p<0.001$ ) in Table 2. The risk of $\mathrm{AA}$ and $\mathrm{AD}$ was higher in patients with HTN, IHD, and stroke than in patients without these comorbidities. As shown in Figure 2, the cumulative incidence of AA and $\mathrm{AD}$ in subsequent years was higher in the TB cohort than in the non-TB cohort (log-rank test, $p<0.001$ ).

Table 2. Factors of aortic aneurysm and aortic dissection by using Cox regression.

\begin{tabular}{|c|c|c|c|c|c|c|c|c|}
\hline Variables & Crude HR & \multicolumn{2}{|c|}{$95 \%$ CI } & $p$ & aHR & \multicolumn{2}{|c|}{$95 \%$ CI } & $p$ \\
\hline $\begin{array}{l}\text { Without } \\
\text { With }\end{array}$ & $\begin{array}{c}\text { Reference } \\
2.064\end{array}$ & 1.154 & 2.875 & $<0.001$ & $\begin{array}{c}\text { Reference } \\
1.711\end{array}$ & 1.098 & 2.666 & $<0.001$ \\
\hline $\begin{array}{c}\text { Sex } \\
\text { Male } \\
\text { Female }\end{array}$ & $\begin{array}{c}1.501 \\
\text { Reference }\end{array}$ & 0.905 & 2.408 & 0.110 & $\begin{array}{c}1.440 \\
\text { Reference }\end{array}$ & 0.899 & 2.306 & 0.129 \\
\hline $\begin{array}{l}\text { Age group (years } \\
\begin{array}{c}20-44 \\
45-69 \\
\geq 70\end{array}\end{array}$ & $\begin{array}{c}\text { Reference } \\
1.786 \\
2.065\end{array}$ & $\begin{array}{l}0.772 \\
0.989\end{array}$ & $\begin{array}{l}2.345 \\
3.397\end{array}$ & $\begin{array}{l}0.512 \\
0.668\end{array}$ & $\begin{array}{c}\text { Reference } \\
1.518 \\
1.669\end{array}$ & $\begin{array}{l}0.565 \\
0.789\end{array}$ & $\begin{array}{l}2.103 \\
3.020\end{array}$ & $\begin{array}{l}0.533 \\
0.696\end{array}$ \\
\hline $\begin{array}{c}\text { Insured premium } \\
\text { (NT\$) } \\
<18,000 \\
18,000-34,999 \\
\geq 35,000\end{array}$ & $\begin{array}{c}\text { Reference } \\
0.000 \\
0.000\end{array}$ & - & - & $\begin{array}{l}0.997 \\
0.997\end{array}$ & $\begin{array}{c}\text { Reference } \\
0.000 \\
0.000\end{array}$ & - & - & $\begin{array}{l}0.998 \\
0.998\end{array}$ \\
\hline $\begin{array}{c}\text { DM } \\
\text { Without } \\
\text { With } \\
\text { HTN }\end{array}$ & $\begin{array}{c}\text { Reference } \\
1.589\end{array}$ & 1.062 & 2.267 & 0.020 & $\begin{array}{c}\text { Reference } \\
1.336\end{array}$ & 0.828 & 2.154 & 0.236 \\
\hline $\begin{array}{l}\text { Without } \\
\text { With }\end{array}$ & $\begin{array}{c}\text { Reference } \\
2.309\end{array}$ & 1.498 & 3.020 & $<0.001$ & $\begin{array}{c}\text { Reference } \\
1.471\end{array}$ & 1.311 & 1.711 & $<0.001$ \\
\hline $\begin{array}{l}\text { Hyperlipidemia } \\
\text { Without } \\
\text { With } \\
\text { IHD }\end{array}$ & $\begin{array}{c}\text { Reference } \\
0.000\end{array}$ & - & - & 0.972 & $\begin{array}{c}\text { Reference } \\
0.000\end{array}$ & - & - & 0.786 \\
\hline $\begin{array}{l}\text { Without } \\
\text { With } \\
\text { COPD }\end{array}$ & $\begin{array}{c}\text { Reference } \\
1.903\end{array}$ & 1.564 & 2.303 & $<0.001$ & $\begin{array}{c}\text { Reference } \\
1.825\end{array}$ & 1.555 & 2.225 & $<0.001$ \\
\hline $\begin{array}{l}\text { Without } \\
\text { With } \\
\text { Stroke }\end{array}$ & $\begin{array}{c}\text { Reference } \\
0.986\end{array}$ & 0.387 & 1.567 & 0.084 & $\begin{array}{c}\text { Reference } \\
1.087\end{array}$ & 0.460 & 1.626 & 0.067 \\
\hline $\begin{array}{l}\text { Without } \\
\text { With } \\
\text { CKD }\end{array}$ & $\begin{array}{c}\text { Reference } \\
2.976\end{array}$ & 1.876 & 4.021 & $<0.001$ & $\begin{array}{c}\text { Reference } \\
2.027\end{array}$ & 1.690 & 2.529 & $<0.001$ \\
\hline $\begin{array}{l}\text { Without } \\
\text { With } \\
\text { PAOD }\end{array}$ & $\begin{array}{c}\text { Reference } \\
1.768\end{array}$ & 1.001 & 2.897 & 0.050 & $\begin{array}{c}\text { Reference } \\
1.563\end{array}$ & 0.881 & 2.670 & 0.323 \\
\hline $\begin{array}{l}\text { Without } \\
\text { With } \\
\text { Obesity }\end{array}$ & $\begin{array}{c}\text { Reference } \\
0.000\end{array}$ & - & - & 0.913 & $\begin{array}{c}\text { Reference } \\
0.000\end{array}$ & - & - & 0.958 \\
\hline $\begin{array}{l}\text { Without } \\
\text { With }\end{array}$ & $\begin{array}{c}\text { Reference } \\
0.000\end{array}$ & - & - & 0.984 & $\begin{array}{c}\text { Reference } \\
0.000\end{array}$ & - & - & 0.982 \\
\hline $\begin{array}{l}\text { Urbanization leve } \\
\begin{array}{r}1 \text { (The highest) } \\
2 \\
3 \\
4 \text { (The lowest) }\end{array}\end{array}$ & $\begin{array}{c}1.726 \\
1.682 \\
1.201 \\
\text { Reference }\end{array}$ & $\begin{array}{l}1.329 \\
1.345 \\
1.006\end{array}$ & $\begin{array}{l}2.676 \\
2.443 \\
1.703\end{array}$ & $\begin{array}{c}<0.001 \\
<0.001 \\
0.045\end{array}$ & $\begin{array}{c}1.637 \\
1.606 \\
1.186 \\
\text { Reference }\end{array}$ & $\begin{array}{l}1.278 \\
1.259 \\
0.936\end{array}$ & $\begin{array}{l}2.461 \\
2.368 \\
1.694\end{array}$ & $\begin{array}{c}<0.001 \\
<0.001 \\
0.287\end{array}$ \\
\hline
\end{tabular}

$\mathrm{HR}=$ hazard ratio, $\mathrm{CI}=$ confidence interval, $\mathrm{aHR}=$ adjusted hazard ratio: adjusted variables listed in the table, $\mathrm{TB}=\mathrm{tuberculosis,}$ $\mathrm{DM}=$ diabetes mellitus, $\mathrm{HTN}=$ hypertension, $\mathrm{IHD}=$ ischemic heart disease, $\mathrm{COPD}=$ chronic obstructive pulmonary disease, $\mathrm{CKD}=\mathrm{chronic}$ kidney disease, PAOD = peripheral arterial occlusive disease. 


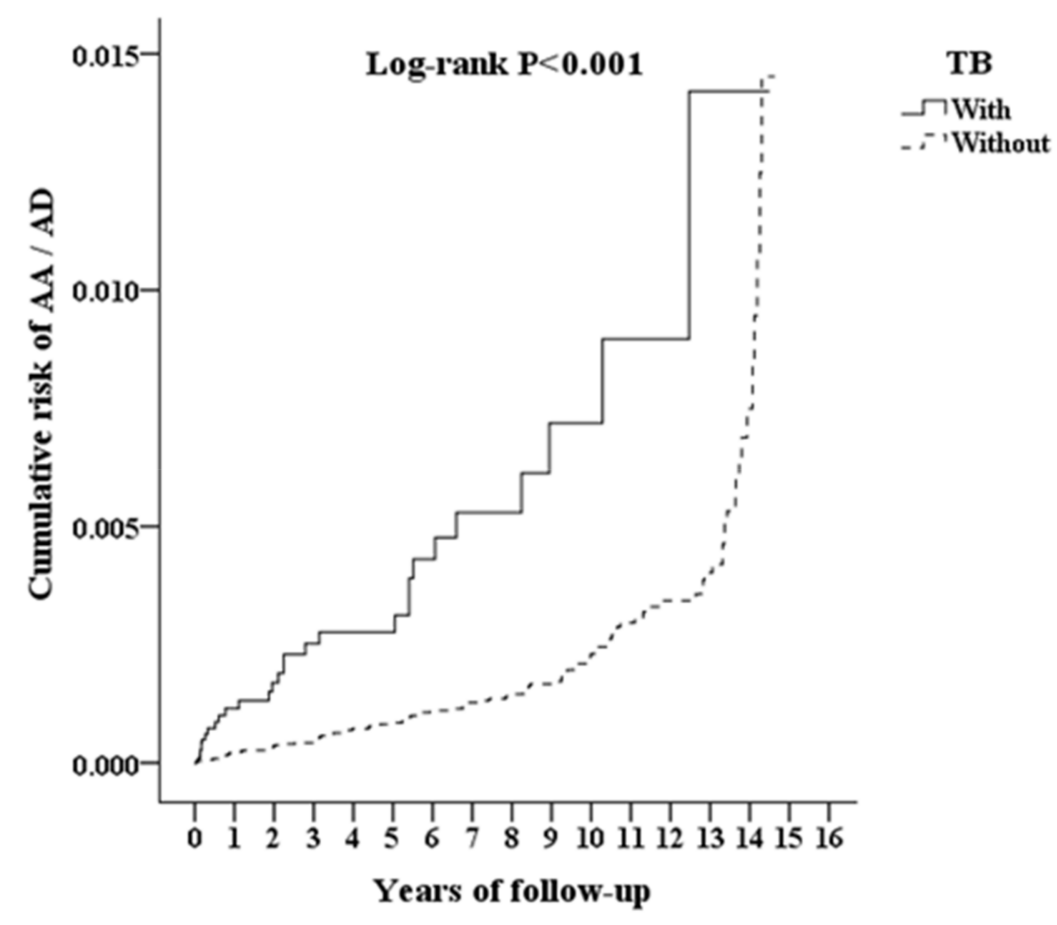

Figure 2. Kaplan-Meier curve for the cumulative risk of aortic aneurysm and aortic dissection due to tuberculosis. $\mathrm{TB}=$ tuberculosis, $\mathrm{AA}=$ aortic aneurysm, $\mathrm{AD}=$ aortic dissection.

Table 3 shows the stratified analysis performed based on demographic factors and comorbidities. The incidence of $\mathrm{AA}$ and $\mathrm{AD}$ was higher in the $\mathrm{TB}$ cohort than in the non- $\mathrm{TB}$ cohort (0.185 vs. 0.15 per 1000 person-years), and the overall IRR of AA and AD was 1.235-fold higher in the $\mathrm{TB}$ cohort than in the non-TB cohort. The sex-specific relative risk of $\mathrm{AA}$ and $\mathrm{AD}$ was higher in patients with $\mathrm{TB}$ than in those without $\mathrm{TB}$ regardless of sex (males, adjusted $\mathrm{HR}=1.738 ; 95 \% \mathrm{CI}=1.115-2.702$; females, adjusted $\mathrm{HR}=1.616$; $95 \% \mathrm{CI}=1.034-2.518)$. The age-specific relative risk of $\mathrm{AA}$ and $\mathrm{AD}$ was higher in patients with TB regardless of age group. The age-specific incidence of $\mathrm{AA}$ and $\mathrm{AD}$ increased in both cohorts, with the highest rate in patients with TB aged $\geq 70$ years $(0.244$ per 1000 person-years). In the comorbidity-specific analysis, the adjusted HR for AA and AD was higher in patients with TB than in those without TB, regardless of the presence of DM, IHD, COPD, stroke, or CKD.

Table 3. Incidence and aHR for aortic aneurysm and aortic dissection in the TB and non-TB cohorts stratified by sex, age, socioeconomic status, and comorbidities.

\begin{tabular}{|c|c|c|c|c|c|c|c|c|c|c|c|c|}
\hline \multirow{2}{*}{$\begin{array}{c}\text { TB } \\
\text { Variables }\end{array}$} & \multicolumn{2}{|c|}{ With } & \multicolumn{2}{|c|}{ Without } & \multicolumn{8}{|c|}{ With vs. without (Reference) } \\
\hline & \multirow{2}{*}{$\begin{array}{c}\text { Event } \\
55\end{array}$} & \multirow{2}{*}{$\begin{array}{c}\begin{array}{c}\text { Rate } \\
\text { (per 10 } \\
\text { PYs) }\end{array} \\
0.185\end{array}$} & \multirow{2}{*}{$\begin{array}{c}\text { Event } \\
94\end{array}$} & \multirow{2}{*}{$\begin{array}{c}\begin{array}{c}\text { Rate } \\
\text { (per 10 } \\
\text { (pYs) }\end{array} \\
0.150\end{array}$} & \multirow{2}{*}{$\begin{array}{l}\text { IRR } \\
1.235\end{array}$} & \multicolumn{2}{|c|}{$95 \%$ CI } & \multirow{2}{*}{$\begin{array}{c}p \\
<0.001\end{array}$} & \multirow{2}{*}{$\begin{array}{l}\text { aHR } \\
1.711\end{array}$} & \multicolumn{2}{|c|}{$95 \%$ CI } & $p$ \\
\hline Overall & & & & & & 1.071 & 1.567 & & & 1.098 & 2.666 & $<0.001$ \\
\hline Sex & & & & & & & & & & & & \\
\hline Male & 43 & 0.202 & 72 & 0.161 & 1.254 & 1.139 & 1.631 & $<0.001$ & 1.738 & 1.115 & 2.702 & $<0.001$ \\
\hline Female & 12 & 0.143 & 22 & 0.123 & 1.165 & 1.032 & 1.868 & 0.006 & 1.616 & 1.034 & 2.518 & 0.008 \\
\hline \multicolumn{13}{|l|}{$\begin{array}{l}\text { Age group } \\
\text { (years) }\end{array}$} \\
\hline $20-44$ & 2 & 0.061 & 3 & 0.051 & 1.188 & 1.046 & 2.976 & 0.002 & 1.645 & 1.051 & 2.553 & 0.002 \\
\hline $45-69$ & 12 & 0.125 & 18 & 0.098 & 1.270 & 1.088 & 2.000 & $<0.001$ & 1.760 & 1.129 & 2.712 & $<0.001$ \\
\hline$\geq 70$ & 41 & 0.244 & 73 & 0.189 & 1.287 & 1.148 & 1.669 & $<0.001$ & 1.784 & 1.153 & 2.789 & $<0.001$ \\
\hline
\end{tabular}


Table 3. Cont.

\begin{tabular}{|c|c|c|c|c|c|c|c|c|c|c|c|c|}
\hline \multirow[b]{2}{*}{ Variables } & \multicolumn{2}{|c|}{ With } & \multicolumn{2}{|c|}{ Without } & \multicolumn{8}{|c|}{ With vs. without (Reference) } \\
\hline & Event & $\begin{array}{c}\text { Rate } \\
\text { (per } 10^{3} \\
\text { PYs) }\end{array}$ & Event & $\begin{array}{c}\text { Rate } \\
\text { (per 10 } \\
\text { PYs) }\end{array}$ & IRR & \multicolumn{2}{|c|}{$95 \% \mathrm{CI}$} & $p$ & aHR & \multicolumn{2}{|c|}{$95 \%$ CI } & $p$ \\
\hline \multicolumn{13}{|l|}{$\begin{array}{l}\text { Insured } \\
\text { premium } \\
\text { (NT\$) }\end{array}$} \\
\hline$<18,000$ & 55 & 0.188 & 94 & 0.152 & 1.238 & 1.178 & 1.571 & $<0.001$ & 1.711 & 1.098 & 2.666 & $<0.001$ \\
\hline $18,000-34,999$ & 0 & 0.000 & 0 & 0.000 & - & - & - & - & - & - & - & - \\
\hline$\geq 35,000$ & 0 & 0.000 & 0 & 0.000 & - & - & - & - & - & - & - & - \\
\hline \multicolumn{13}{|l|}{$\mathrm{DM}$} \\
\hline Without & 40 & 0.204 & 73 & 0.169 & 1.208 & 1.070 & 1.594 & 0.003 & 1.672 & 1.074 & 2.609 & 0.001 \\
\hline With & 15 & 0.149 & 21 & 0.108 & 1.374 & 1.218 & 2.036 & $<0.001$ & 1.911 & 1.223 & 2.972 & $<0.001$ \\
\hline \multicolumn{13}{|l|}{ HTN } \\
\hline Without & 22 & 0.141 & 39 & 0.139 & 1.018 & 1.006 & 1.541 & 0.036 & 1.411 & 0.995 & 2.194 & 0.053 \\
\hline With & 33 & 0.234 & 55 & 0.159 & 1.468 & 1.347 & 1.899 & $<0.001$ & 2.034 & 1.314 & 3.287 & $<0.001$ \\
\hline \multicolumn{13}{|l|}{ Hyperlipidemia } \\
\hline Without & 55 & 0.200 & 94 & 0.172 & 1.163 & 1.057 & 1.747 & $<0.001$ & 1.711 & 1.098 & 2.666 & $<0.001$ \\
\hline With & 0 & 0.000 & 0 & 0.000 & - & - & - & - & - & - & - & - \\
\hline \multicolumn{13}{|l|}{ IHD } \\
\hline Without & 35 & 0.164 & 52 & 0.131 & 1.257 & 1.105 & 1.685 & $<0.001$ & 1.672 & 1.092 & 2.585 & $<0.001$ \\
\hline With & 20 & 0.237 & 42 & 0.183 & 1.296 & 1.198 & 1.828 & $<0.001$ & 1.797 & 1.153 & 2.801 & $<0.001$ \\
\hline \multicolumn{13}{|l|}{ COPD } \\
\hline Without & 31 & 0.192 & 68 & 0.154 & 1.249 & 1.098 & 1.674 & $<0.001$ & 1.633 & 1.065 & 2.598 & 0.003 \\
\hline With & 24 & 0.177 & 26 & 0.141 & 1.256 & 1.125 & 1.810 & $<0.001$ & 1.740 & 1.117 & 2.712 & $<0.001$ \\
\hline \multicolumn{13}{|l|}{ Stroke } \\
\hline Without & 37 & 0.162 & 57 & 0.130 & 1.242 & 1.069 & 1.655 & $<0.001$ & 1.666 & 1.004 & 2.635 & 0.045 \\
\hline With & 18 & 0.264 & 37 & 0.196 & 1.348 & 1.199 & 1.911 & $<0.001$ & 1.869 & 1.118 & 3.012 & $<0.001$ \\
\hline \multicolumn{13}{|l|}{ CKD } \\
\hline Without & 50 & 0.178 & 88 & 0.148 & 1.202 & 1.104 & 1.549 & $<0.001$ & 1.675 & 1.068 & 2.596 & 0.007 \\
\hline With & 5 & 0.302 & 6 & 0.179 & 1.681 & 1.297 & 2.866 & $<0.001$ & 2.330 & 1.486 & 3.631 & $<0.001$ \\
\hline \multicolumn{13}{|l|}{ PAOD } \\
\hline Without & 55 & 0.185 & 94 & 0.150 & 1.234 & 1.211 & 1.581 & $<0.001$ & 1.711 & 1.098 & 2.666 & $<0.001$ \\
\hline With & 0 & 0.000 & 0 & 0.000 & - & - & - & - & - & - & - & - \\
\hline \multicolumn{13}{|l|}{ Obesity } \\
\hline Without & 55 & 0.185 & 94 & 0.150 & 1.234 & 1.194 & 1.566 & $<0.001$ & 1.711 & 1.098 & 2.666 & $<0.001$ \\
\hline With & 0 & 0.000 & 0 & 0.000 & - & - & - & - & - & - & - & - \\
\hline \multicolumn{13}{|l|}{$\begin{array}{c}\text { Urbanization } \\
\text { level }\end{array}$} \\
\hline $\begin{array}{c}1 \text { (The } \\
\text { highest) }\end{array}$ & 13 & 0.163 & 20 & 0.113 & 1.441 & 1.115 & 2.139 & $<0.001$ & 1.997 & 1.297 & 3.121 & $<0.001$ \\
\hline 2 & 14 & 0.110 & 25 & 0.087 & 1.263 & 1.096 & 1.917 & 0.001 & 1.750 & 1.125 & 2.784 & $<0.001$ \\
\hline 3 & 5 & 0.220 & 10 & 0.210 & 1.048 & 0.964 & 2.121 & 0.067 & 1.452 & 0.917 & 2.266 & 0.154 \\
\hline 4 (The lowest) & 23 & 0.341 & 39 & 0.337 & 1.011 & 0.646 & 1.526 & 0.294 & 1.400 & 0.896 & 2.101 & 0.297 \\
\hline
\end{tabular}

PYs = person-years, aHR = adjusted hazard ratio: adjusted for the variables listed in Table 2, $\mathrm{CI}=$ confidence interval, $\mathrm{IRR}=$ incidence rate ratio, $\mathrm{TB}=$ tuberculosis, $\mathrm{DM}=$ diabetes mellitus, $\mathrm{HTN}=$ hypertension, $\mathrm{IHD}=$ ischemic heart disease, $\mathrm{COPD}=$ chronic obstructive pulmonary disease, $\mathrm{CKD}=$ chronic kidney disease, $\mathrm{PAOD}=$ peripheral arterial occlusive disease.

We categorized the TB cohort into pulmonary, extrapulmonary, and miliary TB subgroups based on ICD-9-CM codes. Table 4 shows the incidence and adjusted HR of AA and $\mathrm{AD}$ for the different types of TB. All TB subgroups had a higher risk of developing AA and AD than patients without $\mathrm{TB}$ (pulmonary: adjusted $\mathrm{HR}=1.561,95 \% \mathrm{CI}=1.005-2.431$; extrapulmonary: adjusted $\mathrm{HR}=1.892,95 \% \mathrm{CI}=1.214-2.936$; and miliary: adjusted $\mathrm{HR}=8.334$, $95 \%$ CI $=5.348-12.896$ ). 
Table 4. Incidence and aHR for aortic aneurysm and aortic dissection in the TB and non-TB cohorts stratified by TB type.

\begin{tabular}{|c|c|c|c|c|c|c|c|c|c|c|c|c|}
\hline \multirow[b]{2}{*}{ TB Subgroup } & \multicolumn{2}{|c|}{ With } & \multicolumn{2}{|c|}{ Without } & \multicolumn{8}{|c|}{ With vs. without (Reference) } \\
\hline & Event & $\begin{array}{c}\text { Rate } \\
\text { (per } 10^{3} \\
\text { PYs) }\end{array}$ & Event & $\begin{array}{c}\text { Rate } \\
\text { (per } 10^{3} \\
\text { PYs) }\end{array}$ & IRR & & & $p$ & aHR & $95^{\circ}$ & CI & $p$ \\
\hline Pulmonary TB & 42 & 0.169 & 94 & 0.150 & 1.126 & 1.058 & 1.490 & 0.001 & 1.561 & 1.005 & 2.431 & 0.044 \\
\hline $\begin{array}{c}\text { Extrapulmonary } \\
\text { TB }\end{array}$ & 9 & 0.205 & 94 & 0.150 & 1.365 & 1.160 & 2.049 & $<0.001$ & 1.892 & 1.214 & 2.936 & $<0.001$ \\
\hline Miliary TB & 4 & 0.902 & 94 & 0.150 & 6.013 & 4.870 & 8.802 & $<0.001$ & 8.334 & 5.348 & 12.896 & $<0.001$ \\
\hline
\end{tabular}

PYs = person-years, aHR = adjusted hazard ratio: adjusted for the variables listed in Table 2; CI = confidence interval, IRR = incidence rate ratio, $\mathrm{TB}=$ tuberculosis.

We conducted a subgroup analysis of the incidence and adjusted HR of the different sites of AA and AD. The adjusted HR was higher in the TB cohort than in the non-TB cohort for $\mathrm{AA}$ and $\mathrm{AD}$ occurring in the thoracic (adjusted $\mathrm{HR}=1.615 ; 95 \% \mathrm{CI}=1.044-2.511$ ), abdominal (adjusted HR $=1.588 ; 95 \% \mathrm{CI}=1.025-2.469$ ), thoracoabdominal (adjusted $\mathrm{HR}=2.910 ; 95 \% \mathrm{CI}=1.876-4.557)$, and unspecified sites (adjusted $\mathrm{HR}=1.823 ; 95 \%$ $\mathrm{CI}=1.175-2.843$ ) (Table 5).

Table 5. Incidence and aHR for aortic aneurysm and aortic dissection stratified by different sites.

\begin{tabular}{ccccccccccccc}
\hline TB & \multicolumn{2}{c}{ With } & \multicolumn{2}{c}{ Without } & \multicolumn{5}{c}{ With vs. without (Reference) } \\
\hline $\begin{array}{c}\text { AA/AD } \\
\text { Subgroup }\end{array}$ & Event & $\begin{array}{c}\text { Rate } \\
\text { (per 10 } \\
\text { PYs) }\end{array}$ & Event & $\begin{array}{c}\text { Rate } \\
\text { (per 10 } \\
\text { PYs) }\end{array}$ & IRR & 95\% CI & p & aHR & 95\% CI & $p$ \\
\hline Overall & 55 & 0.185 & 94 & 0.150 & 1.235 & 1.071 & 1.567 & $<0.001$ & 1.711 & 1.098 & 2.666 & $<0.001$ \\
\hline Thoracic & 16 & 0.054 & 29 & 0.046 & 1.164 & 1.039 & 1.498 & 0.015 & 1.615 & 1.044 & 2.511 & 0.007 \\
Abdominal & 13 & 0.044 & 24 & 0.038 & 1.143 & 1.015 & 1.435 & 0.038 & 1.588 & 1.025 & 2.469 & 0.024 \\
Thoracoabdominal & 1 & 0.003 & 1 & 0.002 & 2.110 & 1.753 & 2.525 & $<0.001$ & 2.910 & 1.876 & 4.557 & $<0.001$ \\
Unspecified site & 25 & 0.084 & 40 & 0.064 & 1.319 & 1.188 & 1.682 & $<0.001$ & 1.823 & 1.175 & 2.843 & $<0.001$ \\
\hline
\end{tabular}

PYs $=$ person-years, aHR $=$ adjusted hazard ratio: adjusted for the variables listed in Table $2, \mathrm{CI}=$ confidence interval, $\mathrm{IRR}=$ incidence rate ratio, $\mathrm{TB}=$ tuberculosis, $\mathrm{AA}=$ aortic aneurysm, $\mathrm{AD}=$ aortic dissection.

Table 6 presents the incidence and adjusted HR of AA and AD in both cohorts stratified by the follow-up period. The adjusted HR was higher in the TB cohort than in the non-TB cohort for all follow-up period brackets (within 6 months: adjusted HR $=6.896$, $95 \% \mathrm{CI}=5.010-8.226 ; 6-12$ months: adjusted $\mathrm{HR}=2.671,95 \% \mathrm{CI}=1.675-3.145 ;$ and $1-5$ years: adjusted $\mathrm{HR}=2.371,95 \% \mathrm{CI}=1.486-2.884)$.

Table 6. Incidence and aHR for aortic aneurysm and aortic dissection stratified by follow-up period.

\begin{tabular}{|c|c|c|c|c|c|c|c|c|c|c|c|c|}
\hline \multirow{3}{*}{$\begin{array}{c}\begin{array}{c}\text { Follow-Up } \\
\text { Period }\end{array} \\
<6 \text { months }\end{array}$} & \multicolumn{2}{|c|}{ With } & \multicolumn{2}{|c|}{ Without } & \multicolumn{8}{|c|}{ With vs. without (Reference) } \\
\hline & \multirow{2}{*}{$\begin{array}{c}\text { Event } \\
17\end{array}$} & \multirow{2}{*}{$\begin{array}{c}\begin{array}{c}\text { Rate } \\
\text { (per 10 } \\
\text { PYs) }\end{array} \\
1.192\end{array}$} & \multirow{2}{*}{$\begin{array}{c}\text { Event } \\
7\end{array}$} & \multirow{2}{*}{$\begin{array}{c}\begin{array}{c}\text { Rate } \\
\text { (per 10 } \\
\text { PYs) }\end{array} \\
\text { PYs) } \\
0.236\end{array}$} & \multirow{2}{*}{$\begin{array}{c}\text { IRR } \\
5.061\end{array}$} & \multicolumn{2}{|c|}{$95 \% \mathrm{CI}$} & \multirow{2}{*}{$\begin{array}{c}p \\
<0.001\end{array}$} & \multirow{2}{*}{$\begin{array}{l}\text { aHR } \\
6.896\end{array}$} & \multicolumn{2}{|c|}{$95 \%$ CI } & $p$ \\
\hline & & & & & & 4.267 & 7.106 & & & 5.010 & 8.226 & $<0.001$ \\
\hline $\begin{array}{c}6 \text { months }-12 \\
\text { months }\end{array}$ & 4 & 0.283 & 5 & 0.169 & 1.671 & 1.335 & 1.986 & $<0.001$ & 2.671 & 1.675 & 3.145 & $<0.001$ \\
\hline $1-5$ years & 20 & 0.136 & 28 & 0.092 & 1.481 & 1.198 & 1.875 & $<0.001$ & 2.371 & 1.486 & 2.884 & $<0.001$ \\
\hline$>5$ years & 14 & 0.115 & 54 & 0.206 & 0.560 & 0.042 & 0.989 & 0.041 & 1.276 & 0.375 & 1.790 & 0.385 \\
\hline
\end{tabular}

PYs = person-years, aHR = adjusted hazard ratio: adjusted for the variables listed in Table $2, \mathrm{CI}=$ confidence interval, $\mathrm{IRR}=$ incidence rate ratio, $\mathrm{TB}=$ tuberculosis. 


\section{Discussion}

This is the first nationwide, population-based cohort study to investigate the risk of $\mathrm{AA}$ and $\mathrm{AD}$ in patients with $\mathrm{TB}$ by subgroup analyses. The overall finding was that patients with $\mathrm{TB}$, overwhelmingly, have an increased risk and incidence of $\mathrm{AA}$ and $\mathrm{AD}$ compared with patients without TB, regardless of sex, age, socioeconomic status, and comorbidities. These findings strengthen the possibility of TB being an independent factor for AA and AD. Widespread hematogenous dissemination of M. tuberculosis (miliary TB) can affect multiple organs, such as the lungs, liver, spleen, and central nervous system. Life-threatening cardiovascular complications have been identified in patients with miliary TB [24]. A previous study showed that disseminated TB had a higher serum procalcitonin level than non-disseminated TB $(0.75 \pm 0.79$ versus $0.14 \pm 0.39 \mathrm{ng} / \mathrm{mL} ; p<0.0001)$, which was correlated with the severity of inflammation and poor prognosis [25]. In our study, compared with patients without TB, patients in the subgroup with miliary TB had the highest adjusted HR of AA and AD (adjusted HR, 8.334; 95\% CI, 5.348-12.896), which highlighted the role of systemic inflammation in the development of AA and AD.

Tuberculous mycotic AAs can develop through direct invasion of the aortic intima, seeding adventitia from the vasa vasorum, and most commonly, direct extension from an adjacent focus of TB infection [26]. In addition to direct aortic damage by M. tuberculosis, we propose two possible mechanisms of how TB causes AA and AD. First, TB infection could increase the expression of matrix metalloproteinases (MMPs) via multiple intracellular signaling cascades, mainly NF- $\mathrm{KB}, \mathrm{p} 38$, and the mitogen-activated protein kinase pathway, and reflect the disease severity $[27,28]$. MMPs are zinc-dependent endopeptidase proteins that can degrade and fragment various components of the extracellular matrix (ECM), such as collagen, elastin, fibronectin, and laminin. $\mathrm{AA}$ and $\mathrm{AD}$ occur as a result of disruption of the aortic wall integrity caused by ECM degradation. MMP-1, MMP-2, and MMP-9 have been shown to be fundamental in the development of AA and AD [29,30]. Previous studies showed that high plasma MMP-9 levels are associated with increased AA and AD formation and especially aneurysm rupture [22,31,32]. Hence, elevated MMP levels may contribute to TB-induced AA and AD.

Second, atherosclerosis may cause mechanical weakening of the aortic wall, compensatory lumen enlargement, and activation of inflammation-inducing proteolytic enzymes, resulting in AA formation $[33,34]$. Furthermore, an ulcerating atherosclerotic plaque that penetrates through the elastic lamina into the media can cause intramural hematoma, dissection, or rupture $[35,36]$. Various microbes may contribute to atherosclerotic processes, including Helicobacter pylori, Chlamydia pneumoniae, cytomegalovirus, hepatitis $\mathrm{C}$ virus, and human immunodeficiency virus [37,38]. M. tuberculosis may also participate in the development of atherosclerosis. TB infection and atherosclerosis share similar inflammatory processes, which involve increased expression of proinflammatory cytokines (interleukin [IL]-1, IL-2, IL-6, tumor necrosis factor- $\alpha$, and interferon- $\gamma$ ) and the activation of immune cells (monocytes, macrophage, CD4 ${ }^{+}$T helper 1 [TH1] cells, and TH17 cells) [39]. Previous studies have provided convincing evidence that antibodies against mycobacterial heatshock protein 65 induce the development of atherosclerosis [40,41]. These findings suggest the involvement of $M$. tuberculosis in atherosclerotic processes that lead to the development of $\mathrm{AA}$ and $\mathrm{AD}$.

Ascertainment bias in this study should be considered because patients with TB may be more likely to be diagnosed with AA and AD because of additional chest imaging studies for the workup of TB. To overcome this bias, we conducted stratification analyses of the follow-up periods and the location sites of $\mathrm{AA}$ and $\mathrm{AD}$. We found a prolonged risk of $\mathrm{AA}$ and $\mathrm{AD}$ up to the 5th year after diagnosis. We also found that patients with TB had higher adjusted HRs in both thoracic and abdominal regions than the control participants. These findings confirm that the increased risk of $\mathrm{AA}$ and $\mathrm{AD}$ in patients with $\mathrm{TB}$ is not caused by imaging studies bias.

This study has some limitations. First, NHIRD does not record family history and health-related lifestyle factors in detail, including smoking, body mass index, and alcohol 
consumption, which were potential confounding factors in this study. Second, relevant clinical variables, including imaging results, acid-fast staining, and culture reports, were unavailable from the database; therefore, we could not confirm the time of TB smear or culture-negativity or assess the severity of TB. Third, the Taiwan Centers for Disease Control did not widely implement latent TB infection screening and treatment until 2016, especially for all age groups with a history of close contact with patients with TB. All cases included in our study between 2000 and 2015 were of active TB infection; therefore, we could not investigate the risk of $\mathrm{AA}$ and $\mathrm{AD}$ in patients with latent $\mathrm{TB}$. Fourth, bias caused by unknown confounders remains in this retrospective cohort study despite meticulous adjustments. Therefore, a well-designed randomized prospective control study is warranted to conclusively establish a causal relationship between $\mathrm{TB}$ and the subsequent development of AA and AD.

\section{Conclusions}

We conducted a nationwide population-based cohort study to investigate the association between $\mathrm{TB}$ and the development of $\mathrm{AA}$ and $\mathrm{AD}$ and analyzed the risk of $\mathrm{AA}$ and $A D$ in TB subgroups. Patients with TB had a 1.711-fold higher risk of developing $\mathrm{AA}$ and $\mathrm{AD}$ than patients without $\mathrm{TB}$, particularly in the miliary $\mathrm{TB}$ subgroup. These findings strengthen the association of systemic inflammation between these two diseases. Physicians should consider the subsequent development of AA and AD while treating patients with TB.

Author Contributions: Conceptualization, M.-T.C. and C.-H.S.; methodology, C.-H.C. and W.-C.C.; software, C.-H.C. and W.-C.C.; formal analysis, M.-T.C., C.-H.C., W.-C.C. and C.-H.S.; resources, W.-C.C.; data curation, M.-T.C., C.-K.P. and H.-Y.K.; writing-original draft preparation, M.-T.C.; writing-review and editing, M.-T.C., H.-Y.K., C.-K.P., W.-C.C. and C.-H.S.; visualization, M.-T.C.; supervision, W.-C.C. and C.-H.S. All authors have read and agreed to the published version of the manuscript.

Funding: This study was supported by grants from the Tri-Service General Hospital (TSGH-B-110012).

Institutional Review Board Statement: The study was conducted according to the guidelines of the Declaration of Helsinki and approved by the Institutional Review Board of Tri-Service General Hospital, National Defense Medical Center (TSGHIRB No. B-100-21).

Informed Consent Statement: Patient consent was waived due to all personal data in National Health Insurance Research Database had been multiply encrypted.

Data Availability Statement: Restrictions apply to the availability of these data. Data was obtained from National Health Insurance database and are available from the authors with the permission of National Health Insurance Administration of Taiwan.

Conflicts of Interest: The authors declare no conflict of interest.

\section{References}

1. World Health Organization. Global Tuberculosis Report; World Health Organization: Geneva, Switzerland, 2020.

2. Centers for Disease Control. Taiwan Tuberculosis Control Report 2019; Ministry of Health and Welfare: Taipei, Taiwan, 2020.

3. Ravimohan, S.; Kornfeld, H.; Weissman, D.; Bisson, G.P. Tuberculosis and lung damage: From epidemiology to pathophysiology. Eur. Respir. Rev. 2018, 27, 170077. [CrossRef] [PubMed]

4. Ramírez-Lapausa, M.; Menéndez-Saldaña, A.; Noguerado-Asensio, A. Extrapulmonary tuberculosis. Rev. Esp. Sanid. Penit. 2015, 17, 3-11. [CrossRef] [PubMed]

5. Davis, F.M.; Daugherty, A.; Lu, H.S. Updates of Recent Aortic Aneurysm Research. Arterioscler. Thromb. Vasc. Biol. 2019, 39, e83-e90. [CrossRef] [PubMed]

6. Gawinecka, J.; Schönrath, F.; von Eckardstein, A. Acute aortic dissection: Pathogenesis, risk factors and diagnosis. Swiss Med. Wkly. 2017, 147, w14489.

7. Yeh, T.-Y.; Chen, C.-Y.; Huang, J.-W.; Chiu, C.-C.; Lai, W.-T.; Huang, Y.-B. Epidemiology and Medication Utilization Pattern of Aortic Dissection in Taiwan: A Population-Based Study. Medicine 2015, 94, e1522. [CrossRef]

8. Braverman, A.C. Acute aortic dissection: Clinician update. Circulation 2010, 122, 184-188. [CrossRef]

9. Keisler, B.; Carter, C. Abdominal aortic aneurysm. Am. Fam. Physician 2015, 91, 538-543. 
10. Nienaber, C.A.; Powell, J.T. Management of acute aortic syndromes. Eur. Heart J. 2012, 33, 26-35. [CrossRef]

11. Osler, W. The Gulstonian Lectures, on Malignant Endocarditis. Br. Med. J. 1885, 1, 467-470. [CrossRef]

12. Sörelius, K.; Budtz-Lilly, J.; Mani, K.; Wanhainen, A. Systematic Review of the Management of Mycotic Aortic Aneurysms. Eur. J. Vasc. Endovasc. Surg. 2019, 58, 426-435. [CrossRef]

13. Peng, Y.C.; Lin, C.L.; Hsu, W.Y.; Chang, C.S.; Yeh, H.Z.; Kao, C.H. Risk of liver cirrhosis in patients with tuberculosis: A nationwide cohort study. Eur. J. Clin. Investig. 2015, 45, 663-669. [CrossRef]

14. Shen, C.H.; Chou, C.H.; Liu, F.C.; Lin, T.Y.; Huang, W.Y.; Wang, Y.C.; Kao, C.H. Association Between Tuberculosis and Parkinson Disease: A Nationwide, Population-Based Cohort Study. Medicine 2016, 95, e2883. [CrossRef]

15. Wang, S.H.; Chung, C.H.; Huang, T.W.; Tsai, W.C.; Peng, C.K.; Huang, K.L.; Perng, W.C.; Chian, C.F.; Chien, W.C.; Shen, C.H. Bidirectional association between tuberculosis and sarcoidosis. Respirology 2019, 24, 467-474. [CrossRef]

16. Lin, Y.C.; Liang, S.J.; Liu, Y.H.; Hsu, W.H.; Shih, C.M.; Sung, F.C.; Chen, W. Tuberculosis as a risk factor for systemic lupus erythematosus: Results of a nationwide study in Taiwan. Rheumatol. Int. 2012, 32, 1669-1673. [CrossRef]

17. Chung, W.S.; Lin, C.L.; Hung, C.T.; Chu, Y.H.; Sung, F.C.; Kao, C.H.; Yeh, J.J. Tuberculosis increases the subsequent risk of acute coronary syndrome: A nationwide population-based cohort study. Int. J. Tuberc. Lung Dis. 2014, 18, 79-83. [CrossRef]

18. Wang, S.H.; Chien, W.C.; Chung, C.H.; Lin, F.H.; Peng, C.K.; Chian, C.F.; Shen, C.H. Tuberculosis increases the risk of peripheral arterial disease: A nationwide population-based study. Respirology 2017, 22, 1670-1676. [CrossRef]

19. Kaufmann, S.H.; Dorhoi, A. Inflammation in tuberculosis: Interactions, imbalances and interventions. Curr. Opin. Immunol. 2013, 25, 441-449. [CrossRef]

20. Galkina, E.; Ley, K. Immune and inflammatory mechanisms of atherosclerosis (*). Annu. Rev. Immunol. 2009, $27,165-197$. [CrossRef]

21. Libby, P.; Buring, J.E.; Badimon, L.; Hansson, G.K.; Deanfield, J.; Bittencourt, M.S.; Tokgözoğlu, L.; Lewis, E.F. Atherosclerosis Nat. Rev. Dis. Primers 2019, 5, 56. [CrossRef]

22. Wen, D.; Zhou, X.L.; Li, J.J.; Luo, F.; Zhang, L.; Gao, L.G.; Wang, L.P.; Song, L.; Sun, K.; Zou, Y.B.; et al. Plasma concentrations of interleukin-6, C-reactive protein, tumor necrosis factor- $\alpha$ and matrix metalloproteinase-9 in aortic dissection. Clin. Chim. Acta 2012, 413, 198-202. [CrossRef]

23. Lindholt, J.S.; Shi, G.P. Chronic inflammation, immune response, and infection in abdominal aortic aneurysms. Eur. J. Vasc. Endovasc. Surg. 2006, 31, 453-463. [CrossRef] [PubMed]

24. Sharma, S.K.; Mohan, A.; Sharma, A. Challenges in the diagnosis \& treatment of miliary tuberculosis. Indian J. Med. Res. 2012, 135, 703-730. [PubMed]

25. Ugajin, M.; Miwa, S.; Shirai, M.; Ohba, H.; Eifuku, T.; Nakamura, H.; Suda, T.; Hayakawa, H.; Chida, K. Usefulness of serum procalcitonin levels in pulmonary tuberculosis. Eur. Respir. J. 2011, 37, 371. [CrossRef] [PubMed]

26. Long, R.; Guzman, R.; Greenberg, H.; Safneck, J.; Hershfield, E. Tuberculous mycotic aneurysm of the aorta: Review of published medical and surgical experience. Chest 1999, 115, 522-531. [CrossRef]

27. Elkington, P.T.; Ugarte-Gil, C.A.; Friedland, J.S. Matrix metalloproteinases in tuberculosis. Eur. Respir. J. 2011, 38, 456-464. [CrossRef]

28. Sabir, N.; Hussain, T.; Mangi, M.H.; Zhao, D.; Zhou, X. Matrix metalloproteinases: Expression, regulation and role in the immunopathology of tuberculosis. Cell Prolif. 2019, 52, e12649. [CrossRef]

29. Zhang, X.; Shen, Y.H.; LeMaire, S.A. Thoracic aortic dissection: Are matrix metalloproteinases involved? Vascular 2009, 17, 147-157. [CrossRef]

30. Maguire, E.M.; Pearce, S.W.A.; Xiao, R.; Oo, A.Y.; Xiao, Q. Matrix Metalloproteinase in Abdominal Aortic Aneurysm and Aortic Dissection. Pharmaceuticals 2019, 12, 118. [CrossRef]

31. Wilson, W.R.; Anderton, M.; Choke, E.C.; Dawson, J.; Loftus, I.M.; Thompson, M.M. Elevated plasma MMP1 and MMP9 are associated with abdominal aortic aneurysm rupture. Eur. J. Vasc. Endovasc. Surg. 2008, 35, 580-584. [CrossRef]

32. Kurihara, T.; Shimizu-Hirota, R.; Shimoda, M.; Adachi, T.; Shimizu, H.; Weiss, S.J.; Itoh, H.; Hori, S.; Aikawa, N.; Okada, Y. Neutrophil-derived matrix metalloproteinase 9 triggers acute aortic dissection. Circulation 2012, 126, 3070-3080. [CrossRef]

33. Xu, C.; Zarins, C.K.; Glagov, S. Aneurysmal and occlusive atherosclerosis of the human abdominal aorta. J. Vasc. Surg. 2001, 33, 91-96. [CrossRef] [PubMed]

34. Golledge, J.; Norman, P.E. Atherosclerosis and abdominal aortic aneurysm: Cause, response, or common risk factors? Arterioscler. Thromb. Vasc. Biol. 2010, 30, 1075-1077. [CrossRef] [PubMed]

35. Stanson, A.W.; Kazmier, F.J.; Hollier, L.H.; Edwards, W.D.; Pairolero, P.C.; Sheedy, P.F.; Joyce, J.W.; Johnson, M.C. Penetrating atherosclerotic ulcers of the thoracic aorta: Natural history and clinicopathologic correlations. Ann. Vasc. Surg. 1986, 1, 15-23. [CrossRef]

36. Hayashi, H.; Matsuoka, Y.; Sakamoto, I.; Sueyoshi, E.; Okimoto, T.; Hayashi, K.; Matsunaga, N. Penetrating atherosclerotic ulcer of the aorta: Imaging features and disease concept. Radiographics 2000, 20, 995-1005. [CrossRef] [PubMed]

37. Campbell, L.A.; Rosenfeld, M.E. Infection and Atherosclerosis Development. Arch. Med. Res. 2015, 46, 339-350. [CrossRef] [PubMed]

38. Pothineni, N.V.K.; Subramany, S.; Kuriakose, K.; Shirazi, L.F.; Romeo, F.; Shah, P.K.; Mehta, J.L. Infections, atherosclerosis, and coronary heart disease. Eur. Heart J. 2017, 38, 3195-3201. [CrossRef] [PubMed] 
39. Huaman, M.A.; Henson, D.; Ticona, E.; Sterling, T.R.; Garvy, B.A. Tuberculosis and Cardiovascular Disease: Linking the Epidemics. Trop. Dis. Travel Med. Vaccines 2015, 1, 1-7. [CrossRef]

40. Gruber, R.; Lederer, S.; Bechtel, U.; Lob, S.; Riethmüller, G.; Feucht, H.E. Increased antibody titers against mycobacterial heat-shock protein 65 in patients with vasculitis and arteriosclerosis. Int. Arch. Allergy Immunol. 1996, 110, 95-98. [CrossRef]

41. Zhu, J.; Katz, R.J.; Quyyumi, A.A.; Canos, D.A.; Rott, D.; Csako, G.; Zalles-Ganley, A.; Ogunmakinwa, J.; Wasserman, A.G.; Epstein, S.E. Association of serum antibodies to heat-shock protein 65 with coronary calcification levels: Suggestion of pathogentriggered autoimmunity in early atherosclerosis. Circulation 2004, 109, 36-41. [CrossRef] 